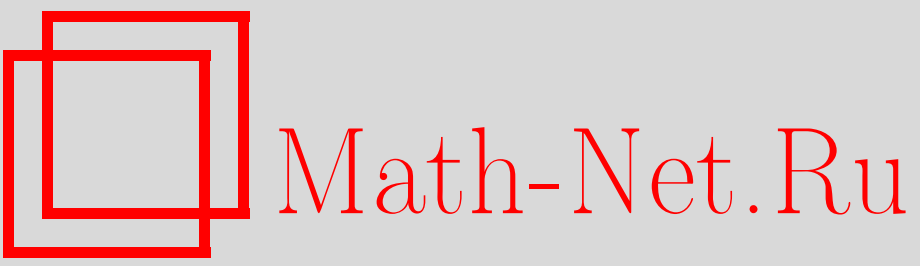

Л. Д. Пустыльников, Доказательство гипотезы квантового хаоса и обобщенные цепные дроби, УМH, 2002, том 57, выпуск 1, 161-162

DOI: https://doi.org/10.4213/rm485

Использование Общероссийского математического портала Math-Net.Ru подразумевает, что вы прочитали и согласны с пользовательским соглашением

http://www.mathnet.ru/rus/agreement

Параметры загрузки:

IP: 3.89 .185 .249

26 апреля 2023 г., 17:37:57 


\title{
ДОКАЗАТЕЛЬСТВО ГИПОТЕЗЫ КВАНТОВОГО ХАОСА И ОБОБЩЕННЫЕ ЦЕПНЫЕ ДРОБИ
}

\author{
Л. Д. Пустыльников
}

В настоящей работе дано доказательство гипотезы квантового хаоса (Г.К.Х.) для широкого класса квантовых систем, содержащего, как частный случай, хорошо известную и популярную модель вращающейся частицы, находящейся под действием $\delta$-толчков ("kicked rotator"), которая во многих работах рассматривалась как один из главных объектов в связи с подтверждением этой гипотезы. Общая постановка Г.К.Х., выдвинутая в [1], состоит в том, что расстояния между соседними уровнями энергии распределены по закону, близкому к закону Пуассона, и до сих пор она не была доказана ни для одной системы. Доказательство Г.К.Х., предложенное здесь (теорема 1), существенно использует результаты о распределении расстояний между соседними дробными частями значений многочлена [2], а оценка остаточного члена (теорема 2) основана на новой теории обобщенных цепных дробей для векторов [3]. Рассмотрим одномерный нелинейный осциллятор, задаваемьй функцией Гамильтона $H=H(\phi, I, t)=H_{0}(I)+H_{1}(\phi, t)$ :

$$
\frac{d \phi}{d t}=\frac{\partial H}{\partial I}=\frac{d H_{0}}{d I}, \quad \frac{d I}{d t}=-\frac{\partial H}{\partial \phi}=-\frac{\partial H_{1}}{\partial \phi},
$$

где $I, \phi$ - переменные “действие-угол”, $t$ - независимая переменная, а функция $H_{1}(\phi, t)$ имеет период $2 \pi$ по $\phi$, период $T>0$ по $t$ и представляется в виде

$$
H_{1}(\phi, t)=F(\phi) \sum_{k=-\infty}^{\infty} \delta(t-k T),
$$

где $F(\phi)$ - гладкая $2 \pi$-периодическая функция, $\delta=\delta(t)$ - дельта-функция, а суммирование распространено на все целше числа $k$. Здесь же мы предполагаем, что $H_{0}(I)=\sum_{s=0}^{n} b_{s} I^{s}$ - многочлен степени $n \geqslant 2 \mathrm{c}$ коэффициентами $b_{s}=a_{s} / \hbar^{s}(s=0, \ldots, n)$, где $\hbar-$ постоянная Планка, а $a_{s}$ - вещественные числа. В частном случае, когда $n=2$, а $F(\phi)=\gamma \cos \phi(\gamma$-константа), система (1) есть "kicked rotator". Введем гильбертово пространство $L^{2}$ комплексных $2 \pi$-периодических функций как пространство состояний квантовой системы и оператор импульса $\widehat{I}=\frac{\hbar}{i} \frac{\partial}{\partial \phi}$. Изменение волновой функции $\Psi=\Psi(\phi, t) \in L^{2}$ описывается уравнением Шрёдингера

$$
i \hbar \frac{\partial}{\partial t} \Psi(\phi, t)=\widehat{H}(t) \Psi(\phi, t)
$$

где оператор $\widehat{H}(t)=\widehat{H}_{0}+\widehat{H}_{1}(t), \widehat{H}_{0}=\sum_{s=0}^{n} b_{s} \widehat{I}^{s}$, а $\widehat{H}_{1}(t)$ есть предел при $\varepsilon \rightarrow 0(\varepsilon>0)$ операторов умножения на функцию $H_{1}^{(\varepsilon)}$, которая получается из функции $H_{1}$ в $(2)$, если $\delta$-функцию заменить на гладкую функцию $\delta_{\varepsilon}$, сосредоточенную на отрезке $[0, \varepsilon]$, интеграл которой равен 1 . Обозначим через $\Psi_{+}(\phi, n T)$ решение уравнения (3) сразу после момента $t=n T$ и введем оператор монодромии $U: \Psi_{+}(\phi, n T) \rightarrow \Psi_{+}(\phi,(n+1) T)$.

Лемма 1. При $k \in \mathbb{Z}$ рассмотрим функцию $e_{k}(\phi)=\exp (i k \phi) \in L^{2}$. Тогда $U e_{k}(\phi)=$ $\lambda_{k}(\phi) e_{k}(\phi)$, где $\lambda_{k}(\phi)=\exp \left(-\frac{i\left(F(\phi)+T \sum_{s=0}^{n} a_{s} k^{s}\right)}{\hbar}\right)$.

ЗАмечАниЕ 1 . Если $F(\phi)=$ const, то $e_{k}(\phi)$ - собственные функции оператора $U$, а $\lambda_{k}(\phi)-$ их собственные значения.

Работа выполнена при частичной поддержке Российского фонда фундаментальных исследований (гранты № 97-01-00268 и № 99-01-01063). 
ОБОЗнАчения. Символ $\{x\}$ есть дробная часть числа $x, \mathbb{T}^{n}-n$-мерньй тор.

ОПредЕлЕниЕ 1. Следуя [4], величина $\beta_{k}(\phi)=\left\{-\frac{\ln \lambda_{k}(\phi)}{2 \pi i}\right\}$ называется $k$-уровнем квазиэнергии системы (3).

ОПРЕДЕЛЕниЕ 2 . Для любого $k \in \mathbb{Z} k$-уровень и $(k+1)$-уровень квазиэнергии назьваются соседними.

ЗАмечАнИЕ 2. Смысл определения 2 состоит в том, что обобщенные собственные функции $e_{k}(\phi)$ и $e_{k+1}(\phi)$ соответствуют квантовым состояниям, между частотами колебаний $\gamma_{k}=k /(2 \pi)$, $\gamma_{k+1}=(k+1) /(2 \pi)$ которых нет других частот состояний $e_{s}(\phi)$ при $s \neq k, k+1$.

Теорема 1. Предположим, что среди чисел $T a_{2} /(2 \pi \hbar), \ldots, T a_{n} /(2 \pi \hbar)$ хотя бь одно число - иррациональное, $0<\sigma \leqslant 1, N$ - натуральное число, а $D_{N}(\phi, \sigma)$ - количество среди чисел $1, \ldots, N$ таких чисел $k$, что $0 \leqslant \beta_{k+1}(\phi)-\beta_{k}(\phi)<\sigma$. Тогда при любом $\phi$ существует предельная функция распределения $P(\sigma)=\lim _{N \rightarrow \infty} D_{N}(\phi, \sigma) / N$, которая имеет вид $P(\sigma)=\sigma-\sigma^{2} / 2$ и отличается от функиии распределения $P_{\Pi}(\sigma)=1-\exp (-\sigma)$ закона Пуассона с плотностью $\exp (-\sigma)$ членами третьего порядка малости относительно $\sigma$.

Доказательство теоремы 1 следует из леммы 1 , определения 1 и доказанных в [2] теоремы 3 и ее следствия.

ОПРедЕлЕнИЕ 3 . Пусть $\alpha$ - вещественное число. Введем отображение $A=A(\alpha)$ пространства $\mathbb{R}^{n}$ такое, что если $x=\left(x_{1}, \ldots, x_{n}\right) \in \mathbb{R}^{n}$, то $A x=x^{\prime}=\left(x_{1}^{\prime}, \ldots, x_{n}^{\prime}\right)$, где

$$
x_{s}^{\prime}=\sum_{j=0}^{n-s}\left(\begin{array}{c}
s+j \\
j
\end{array}\right) x_{s+j}+\left(\begin{array}{c}
n+1 \\
n-s+1
\end{array}\right) \alpha,
$$

$1 \leqslant s \leqslant n,\left(\begin{array}{c}m \\ l\end{array}\right)$ - число сочетаний из $m$ элементов по $l$.

Далее в формулировке теоремы 2 мы используем теорию $(A, \omega)$-цепных дробей для векторов $x \in \mathbb{R}^{n}[3]$. Эти цепные дроби дают однозначное символическое представление любого вектора $x \in \mathbb{R}^{n}$ в виде $x=\left[q^{(0)}, \ldots, q^{(m)}\right]_{A, \omega}$, если $(A, \omega)$-цепная дробь - конечная.

ТЕорема 2. Предположим, что число $\alpha=T a_{n} /(2 \pi(n+1) \hbar)$ - иррациональное, $A=$ $A(\alpha), 0<\sigma \leqslant 1,0<\rho<1 / 2, N$ - натуральное число, $n_{0}=(116-72 \rho) /(8-16 \rho)$, $a^{*}=\left(a_{1}^{*}, \ldots, a_{n}^{*}\right)$ - вектор с координатами $a_{s}^{*}=\left(T a_{s-1}\right) /(2 \pi \hbar s)(s=1, \ldots, n), D_{N}(\phi, \sigma)$ - количество среди чисел $1, \ldots, N$ таких чисел $k$, что $0 \leqslant \beta_{k+1}(\phi)-\beta_{k}(\phi)<\sigma$. Тогда если $n>n_{0}$, то существует множество $\Gamma \subset \mathbb{T}^{n}$ такое, что для почти всех $a=\left(a_{1}, \ldots, a_{n-1}\right) \in \mathbb{R}^{n-1}$ существует вектор $\omega \in \Gamma$ такой, что $(A, \omega)$-цепная дробь вектора $a^{*}$ - конечная, имеет вид

$$
a^{*}=\left[q^{(0)}, \ldots, q^{(\nu)}\right]_{A, \omega},
$$

и для любого $\varepsilon>0$ при всех $N \geqslant 2$

$$
\left|D_{N}(\phi, \sigma) / N-\left(\sigma-\sigma^{2} / 2\right)\right| \leqslant c_{1} \varepsilon+c_{2}\left(c_{3}+\nu\right) /\left(N^{\rho} \varepsilon\right),
$$

где $c_{1}=c_{1}(n), c_{2}=c_{2}(n), c_{3}=c_{3}(n)-$ константь

\section{СПИСОК ЛИТЕРАТУРЫ}

[1] M. V. Berry, M. Tabor // Proc. Roy. Soc. London A. 1977. V. 356. P. 375-394. [2] Л. Д. Пустыльников // УМН. 1999. Т. 54. №6. C. 173-174. [3] L. D. Pustyl'nikov // J. Math. Sci. 1999. V. 95. № 5. Р. 2552-2563. [4] Я. Б. Зельдович // ЖЭТФ. 1966. Т. 51. C. $1492-1495$. 